Artigos Originais

\title{
Baixa escolaridade como fator limitante para 0 combate à anemia entre gestantes
}

\author{
Low educational level as a limiting factor in the fight against anemia in pregnant women
}

Márcia Regina Vitolo ${ }^{1}$, Camile Boscaini ${ }^{2}$, Gisele Ane Bortolini ${ }^{3}$

\section{RESUMO}

Objetivos: avaliar o impacto sobre os níveis de hemoglobina entre gestantes submetidas à suplementação com sulfato ferroso e orientações alimentares.Métodos: foram avaliadas 197 gestantes acompanhadas no pré-natal de uma unidade de saúde. O grupo intervenção foi composto por 105 gestantes com idade gestacional inicial entre a $14^{\mathrm{a}}$ e a $20^{\mathrm{a}}$ semana de gravidez, as quais receberam prescrição de $60 \mathrm{mg}$ de ferro elementar por dia, por meio de sulfato ferroso, orientações alimentares e dosagem de hemoglobina realizada com fotômetro portátil. A partir de 34 semanas gestacionais esse grupo foi reavaliado quanto à hemoglobina e realizado consumo alimentar de freqüência semiquantitativo. O grupo controle foi avaliado transversalmente e era formado por 92 gestantes com idade gestacional superior a 34 semanas. Considerou-se anemia quando a hemoglobina foi inferior a $11 \mathrm{~g} / \mathrm{dL}$ As gestantes de ambos os grupos foram pesadas e medidas, sendo o diagnóstico nutricional determinado pelo IMC pré-gestacional. Foi realizada análise multivariada por meio de regressão logística utilizando modelo hierárquico. Resultados: a prevalência de anemia ao final do terceiro trimestre, no grupo intervenção, foi de $31,6 \%$, e no grupo controle, 26,1\% ( $\mathrm{p}=0,43$ ). $\mathrm{O}$ uso do suplemento de ferro foi referido por $65 \%$ das gestantes do grupo intervenção, sendo que $67,7 \%$ interromperam o uso em algum momento. Os motivos principais foram: esquecimento $(43,2 \%)$ e enjôo e/ou vômito $(27,2 \%)$. Gestantes com escolaridade inferior a 8 anos de estudo apresentaram 3 vezes mais risco na ocorrência de anemia no terceiro trimestre. Conclusões: o uso de sulfato ferroso não mostrou-se associado à menor prevalência de anemia. Os resultados sugerem que são necessárias mudanças estruturais nas condições socioeconômicas para se modificar o quadro atual quanto à anemia ferropriva.

PALAVRAS-CHAVE: Gravidez; Anemia; Sulfato ferroso; Suplementação alimentar; Hemoglobinas/análise; Escolaridade; Resultado de tratamento

\section{ABSTRACT}

Purpose: to evaluate the impact of supplementary ferrous sulfate and dietary counseling on hemoglobin levels in pregnant women. Methods: a total of 197 pregnant women were evaluated during antenatal care at a health center. The treatment group consisted of 105 women who were prescribed $60 \mathrm{mg}$ dietary iron per day, received dietary counseling and had hemoglobin measured by a portable photometer between the 14th and 20th week of pregnancy. The treatment group was reevaluated according to hemoglobin levels and food intake by a semiquantitative food frequency questionnaire after the 34th week of pregnancy. The control group consisted of 92 women in a cross-sectional study, at no less than 34 weeks of pregnancy. Hemoglobin was analyzed by a portable photometer and anemia was defined concentrations of less than $11 \mathrm{~g} / \mathrm{dL}$. All pregnant women had their weight and height measured. Hierarchical logistic regression model was developed for the multivariate analysis. Results: prevalence of anemia at the end of the third trimester was $31.6 \%$ in the treatment group and $26.1 \%$ in the control group ( $p=0.43$ ). Use of the prescribed supplement was reported by $65 \%$ of women in the treatment group, of which $67.7 \%$ interrupted the treatment at some point. Principal reasons for interrupting treatment were forgetting $(43.2 \%)$ and nausea or vomiting $(27.2 \%)$. Risk of anemia in the third trimester was three times higher in women with less than 8 years of schooling. Conclusions: use of ferrous sulfate was not shown to be associated with lower prevalence of anemia. The results suggest that structural changes in socioeconomic conditions are needed in order to alter the current situation regarding iron deficiency anemia.

KEYWORDS: Pregnancy; Anemia; Ferrous sulfate; Supplementary feeding; Hemoglobins/analysis; Educational status; Treatment outcome

Programa de Pós-Graduação em Ciências Médicas - Fundação Faculdade Federal de Ciências Médicas de Porto Alegre - FFFCMPA - Porto Alegre (RS), Brasil; Universidade do Vale do Rio dos Sinos - UNISINOS - São Leopoldo (RS), Brasil.

1 Professor Adjunto do Programa de Pós Graduação em Ciências Médicas da Fundação Faculdade Federal de Ciências Médicas de Porto Alegre FFFCMPA - Porto Alegre (RS), Brasil.

2 Acadêmico de Nutrição da Universidade do Vale do Rio dos Sinos - UNISINOS - São Leopoldo (RS), Brasil.

3 Mestrando do Programa de Pós - Graduação em Ciências Médicas da Fundação Faculdade Federal de Ciências Médicas de Porto Alegre - FFFCMPA - Porto Alegre (RS), Brasil.

Correspondência: Márcia Regina Vitolo

FFFCMPA - Departamento de Saúde Coletiva - Avenida Sarmento Leite, 245 - 90050-170 - Porto Alegre - RS - e-mail: marciavitolo@hotmail.com 


\section{Introdução}

A Organização Mundial de Saúde estima que a prevalência de anemia entre gestantes de países subdesenvolvidos seja de $52,0 \%$ e de $23,0 \%$ nos países desenvolvidos ${ }^{1}$. No Brasil e na maior parte dos países ainda não há quadro consolidado de informações consistentes que permitam definir com a necessária segurança a prevalência de anemia durante a gestação. Os dados disponíveis no Brasil mostram que a prevalência varia de 12,4 a $54,7 \%$ dependendo da idade gestacional, estrato socioeconômico e região ${ }^{2-7}$.

Entre as principais causas da anemia entre gestantes destaca-se o baixo nível socioeconômico, maior número de partos, baixo nivel educacional, idade gestacional mais avançada, reservas inadequadas de ferro, ausência de suplementação de ferro e dietas deficientes em quantidade e qualidade de ferro ${ }^{2,3,8,9}$. A deficiência de ferro durante a gestação aumenta a mortalidade materna fetal e perinatal, além do aumento da prematuridade. Quarenta por cento de todas as mortes perinatais estão associadas à anemia. Resultados favoráveis à gestação ocorrem com menos freqüência entre 30 e $45 \%$ das gestantes anêmicas e seus filhos tem metade das reservas de ferro ${ }^{1}$. A anemia na gestação está intimamente relacionada com o trabalho de parto prematuro e o baixo peso ao nascimento ${ }^{4,10}$, além de pré-eclampsia, aumento no risco de aborto espontâneo e mortalidade materna e perinatal ${ }^{11-15}$.

A suplementação de ferro é universalmente recomendada durante a gestação para corrigir ou prevenir a deficiência de ferro ${ }^{1}$, pois o consumo de ferro proveniente da alimentação não é suficiente para atingir a recomendação atual de 27 miligramas por dia nessa fase do ciclo vital ${ }^{16}$.

A proposta do presente estudo foi avaliar o impacto e a adesão de uma intervenção baseada na prescrição de suplemento de ferro e orientações alimentares nos niveis de hemoglobina de gestantes de uma Unidade Básica de Saúde. Objetivou-se também avaliar a prevalência e os fatores de risco para anemia.

\section{Métodos}

\section{Desenvolvimento do estudo}

Estudo de intervenção desenvolvido na cidade de São Leopoldo-RS, realizado em parceria com as Coordenações Técnicas da Secretaria Municipal de Saúde e dos profissionais de saúde da Unidade Básica de Saúde denominada de Materno-Infantil.
Essa Unidade localiza-se no centro da cidade e é responsável pela demanda de 620 atendimentos/ mês incluindo obstetrícia e exames pré-câncer.

O cálculo do tamanho da amostra baseouse na freqüência de anemia em gestantes não expostas (ao uso de suplemento) de $45 \%$ no grupo controle e estimou diferença de $25 \%$ na freqüência de anemia entre os grupos no terceiro trimestre gestacional. Outros parâmetros para este cálculo foram: poder de $80 \%$ e nivel de confiança de $95 \%$, o que determinou tamanho amostral de 94 gestantes para o grupo controle e 47 para o grupo intervenção. Considerando previsão de perdas no grupo intervenção, 105 gestantes foram recrutadas.

\section{Grupo intervenção}

O grupo intervenção foi formado por gestantes de 14 a 20 semanas gestacionais que procuraram o obstetra para acompanhamento pré-natal e que não apresentavam doenças crônicas ou alguma doença aguda no momento da coleta de dado que pudesse alterar os niveis de hemoglobina. Após o aceite materno para participar do estudo, estudantes de nutrição treinadas realizaram o questionário com as informações sobre os dados socioeconômicos, antecedentes clínicos, uso de suplementos medicamentosos e hábito alimentar, e realizava-se a dosagem de hemoglobina. Todas as gestantes que não tivessem alguma restrição clínica para o uso de suplemento de ferro receberam o suplemento de sulfato ferroso gratuitamente para ser utilizado diariamente na dosagem de $60 \mathrm{mg}$ de ferro elementar. Quando as gestantes já faziam uso de suplemento de ferro a prescrição anterior era mantida. Os valores de hemoglobina foram fornecidos ao médico responsável pelo atendimento que realizava a prescrição ou os encaminhamentos necessários em situações de niveis de hemoglobina inferiores a $9,0 \mathrm{mg} / \mathrm{dL}$. As gestantes foram orientadas quanto às práticas dietéticas pertinentes ao ciclo vital e receberam folder com linguagem acessivel intitulado "Gestos simples que valem uma vida" abordando a importância de algumas práticas alimentares importantes (ex: alimentos fontes de ferro, consumo de água, alimentos fontes de vitamina C, horário das refeições) para a saúde do feto. Quando as gestantes completavam 34 semanas, foram localizadas por telefone para agendamento da segunda etapa do estudo, que foi a avaliação da dosagem de hemoglobina, verificação dos dados de impacto e adesão ao uso do suplemento de ferro, avaliação antropométrica e hábitos alimentares.

\section{Grupo controle}

Para o grupo controle os critérios de seleção da amostra foram de gestantes que estivessem no 
período gestacional igual ou superior a 34 semanas gestacionais e não apresentassem doenças associadas, sendo analisadas transversalmente. As gestantes que compareciam à Unidade Básica de Saúde foram convidadas a participar do estudo e, após o aceite e assinatura do termo de consentimento pelas mesmas, foram submetidas ao questionário que continha informações sobre dados socioeconômicos, antecedentes clínicos, uso de suplementos medicamentosos e hábito alimentar; realizavam-se ainda a dosagem de hemoglobina e a avaliação antropométrica. As gestantes de ambos os grupos receberam folder estimulando o aleitamento materno exclusivo até o sexto mês de vida e orientações dietéticas sobre a introdução adequada da alimentação complementar no primeiro ano de vida da criança, com orientações e sugestões de papas para a alimentação complementar da criança.

\section{Dosagem de hemoglobina}

Para a dosagem de hemoglobina foram coletadas amostras de sangue capilar por meio de microcuvetas descartáveis, utilizadondo o fotômetro portátil (hemoglobinômetro) marca HemoCue ${ }^{\circledR}$. O resultado era obtido imediatamente pela leitura do aparelho utilizado e expresso em decilitros. A dosagem foi realizada uma única vez, porém na presença de hemoglobina $<10 \mathrm{~g} / \mathrm{dL}$ o teste foi repetido e o resultado foi obtido pela média das duas amostras. Para o diagnóstico da anemia nas gestantes no final da gestação, utilizou-se o critério proposto pela $\mathrm{OMS}^{1}$, que define anemia como a concentração de hemoglobina inferior a $<11 \mathrm{~g} / \mathrm{dL}$. Os valores de hemoglobina novamente foram fornecidos ao médico responsável pelo atendimento.

\section{Avaliação antropométrica}

Para avaliação antropométrica das gestantes, o peso foi verificado em balança da digital portátil, com capacidade para $150 \mathrm{~kg}$, da marca Techline ${ }^{\circledR}$. A estatura foi aferida por estadiômetro fixado em parede lisa e sem rodapé. Utilizou-se o índice de massa corpórea (IMC), que é obtido pela divisão do peso (quilogramas) pela altura (metros) ao quadrado, para o diagnóstico do estado nutricional pré-gestacional e no momento da entrevista. Foram consideradas com baixo peso as gestantes que apresentaram IMC pré-gestacional $<19,8 \mathrm{~kg} / \mathrm{m}^{2}$, eutróficas quando o valor estava entre IMC $\geq 19,8$ e $<25 \mathrm{~kg} / \mathrm{m}^{2}$ e com excesso de peso aquelas com IMC maior ou igual a $25 \mathrm{~kg} / \mathrm{m}^{2}$, de acordo com os parâmetros sugeridos pelo IOM para gestantes ${ }^{16}$. Para a análise de associação com o desfecho as gestantes foram classificadas como peso insuficiente (IMC pré-gestacional $<19,8 \mathrm{~kg} / \mathrm{m}^{2}$ ) e peso suficiente (IMC pré-gestacional $\geq 19,8 \mathrm{~kg} / \mathrm{m}^{2}$ ).

\section{Inquérito alimentar}

O padrão de ingestão alimentar das gestantes foi realizado por meio do inquérito de freqüência semiquantitativo de alimentos, e constavam as seguintes opções de freqüência de consumo: diário, semanal, eventual e nunca, sendo que para a primeira opção o consumo foi quantificado. Os alimentos fontes de ferro avaliados foram: carne, figado, feijão, ovos; as fontes de cálcio: leite, queijo e iogurte; as fontes de vitamina A: alimentos amarelos/alaranjados como manga, mamão, cenoura, abóbora; as fontes de ácido fólico: as folhas verdes escuras; e as fontes de vitamina C: limão, laranja, bergamota, tomate e acerola. Atenção especial foi dada ao consumo de pães, biscoitos e massas, uma vez que no Brasil, desde 18 de junho de 2004, todas as farinhas de trigo e milho comercializadas são enriquecidas com ferro e ácido fólico. A determinação é da Agência Nacional de Vigilância Sanitária (ANVISA), por resolução RDC n ${ }^{\circ} 344$. O regulamento prevê que os fabricantes devem adicionar a cada 100 gramas de farinha no mínimo 4,2 $\mathrm{mg}$ de ferro e $150 \mu \mathrm{cg}$ de ácido fólico. Após levantamento de dados considerou-se para esse estudo que são necessárias cerca de 60 gramas de farinha para a preparação de 100 gramas de pão/bolo/biscoitos e massa.

\section{Banco de dados e análise estatística}

Os dados foram compilados no programa Epi-Info, versão 6.4, com dupla digitação e posterior validação. As análises foram realizadas no programa SPSS, versão 11.0. A homogeneidade da amostra quanto à distribuição das variáveis socioeconômicas, demográficas, biológicas e de pré-natal entre os grupos de intervenção e controle foi examinada pelo teste $\chi^{2}$. Esse teste também foi usado para avaliar o impacto do uso do suplemento de ferro sobre a ocorrência do desfecho de interesse: anemia. As estimativas fornecidas pela análise bivariada foram expressas como odds ratio (OR). Para análise dos possiveis determinantes da anemia as gestantes de ambos os grupos foram analisadas conjuntamente. A análise multivariada foi realizada por meio da regressão logística conforme o modelo hierárquico elaborado: nivel 1- variáveis socioeconômicas; nivel 2- variáveis biológicas; nível 3-variáveis dietéticas, uso de suplemento e tabagismo. Utilizou-se o OR como medida de efeito. As variáveis foram mantidas no modelo quando se mostraram associadas com o desfecho, com valor de $\mathrm{p}$ de até 0,20. Foi considerado o valor de $5 \%(\mathrm{p}<0,05)$ como limiar de significância estatística final.

O projeto de pesquisa foi aprovado pelo Comitê de Ética da Universidade do Vale do Rio dos Sinos. 


\section{Resultados}

Foram avaliadas 197 gestantes, sendo que o grupo controle foi composto por 92 gestantes e o grupo de intervenção inicialmente por 105 gestantes, sendo que destas, 76 completaram o estudo. As 29 perdas foram devidas a mudança de endereço (3), aborto (3), ficha preenchida de forma inadequada (2), término da gravidez antes de a segunda avaliação ser realizada (10), não compareceu para segunda avaliação (10) e recusa no momento da segunda avaliação (1).

No início do estudo $36,2 \%$ das gestantes pertencentes ao grupo intervenção $(n=38)$ apresentaram anemia; a idade gestacional média foi de $16,2 \pm 3,3$ semanas. Destas, $24,8 \%$ já usavam algum suplemento de ferro, 38,1\% estavam iniciando o pré-natal no momento da coleta dos dados e 30,5\% estavam na segunda consulta.

A média da idade gestacional no final da gestação, na segunda avaliação, foi de $35,6 \pm 1,9$ semanas para o grupo intervenção e $36,7 \pm 1,8$ semanas no grupo controle. A Tabela 1 apresenta as características gerais das gestantes de ambos os grupos com idade gestacional superior a 34 semanas. A distribuição das variáveis biológicas, sociódemograficas e de pré-natal foi semelhante para os grupos controle e intervenção, 44,6 e 43,3\% das gestantes tinham escolaridade inferior a 8 anos, respectivamente e em torno de $30 \%$ não estavam trabalhando. Das gestantes do grupo intervenção, $27,6 \%$ tinham idade inferior a 20 anos e, no grupo controle, 16,3\%; respectivamente, 14,3 e 13,2\% não possuíam companheiro e a renda familiar total de mais de $70 \%$ das gestantes de ambos os grupos

Tabela 1 - Características sociodemográficas, paridade e tabagismo entre gestantes do grupo controle e intervenção.

\begin{tabular}{|c|c|c|c|c|c|c|}
\hline & \multicolumn{2}{|c|}{ Grupo controle } & \multicolumn{2}{|c|}{ Grupo intervenção } & \multirow{2}{*}{ OR (IC 95\%) } & \multirow{2}{*}{$p$} \\
\hline & $\mathrm{n}$ & $\%$ & $\mathrm{n}$ & $\%$ & & \\
\hline \multicolumn{7}{|l|}{ Escolaridade } \\
\hline$<8$ anos & 41 & 44,6 & 45 & 43,3 & $1,05(0,5-1,8)$ & 0,8 \\
\hline$\geq 8$ anos & 51 & 55,4 & 59 & 56,7 & 1 & \\
\hline \multicolumn{7}{|l|}{ Idade } \\
\hline$<20$ anos & 15 & 16,3 & 29 & 27,6 & $0,51(0,2-1,0)$ & 0,05 \\
\hline$\geq 20$ anos & 77 & 83,7 & 76 & 72,4 & 1 & \\
\hline \multicolumn{7}{|l|}{ Estado civil } \\
\hline Sem companheiro & 12 & 13,2 & 15 & 14,3 & $0,91(0,4-2,0)$ & 0,82 \\
\hline Com companheiro & 79 & 86,8 & 90 & 85,7 & 1 & \\
\hline \multicolumn{7}{|l|}{ Ocupação } \\
\hline Não remunerada & 60 & 65,2 & 73 & 70,2 & $0,79(0,4-1,4)$ & 0,45 \\
\hline Remunerada & 32 & 34,8 & 31 & 29,8 & 1 & \\
\hline \multicolumn{7}{|l|}{ Renda familiar } \\
\hline$<5$ salários mínimos & 70 & 76,1 & 85 & 81,7 & $0,71(0,3-1,4)$ & 0,33 \\
\hline$\geq$ 5salários mínimos & 22 & 23,9 & 19 & 18,3 & 1 & \\
\hline \multicolumn{7}{|c|}{ Concentração por dormitório } \\
\hline$<3$ pessoas & 16 & 17,4 & 21 & 20,0 & 1 & 0,64 \\
\hline$\geq 3$ pessoas & 76 & 82,6 & 84 & 80,0 & $0,84(0,4-1,7)$ & \\
\hline \multicolumn{7}{|l|}{ Primípara } \\
\hline Não & 63 & 68,5 & 61 & 58,1 & $1,56(0,5-1,8)$ & 0,13 \\
\hline Sim & 29 & 31,5 & 44 & 41,9 & 1 & \\
\hline \multicolumn{7}{|l|}{ Número de gestações } \\
\hline$\leq 3$ & 80 & 87,0 & 17 & 16,2 & 1 & 0,53 \\
\hline$>3$ & 12 & 13,0 & 88 & 83,8 & $0,77(0,3-1,7)$ & \\
\hline \multicolumn{7}{|l|}{ Tabagismo } \\
\hline Sim & 14 & 15,2 & 13 & 12,5 & $0,71(0,3-1,5)$ & 0,58 \\
\hline Não & 78 & 84,8 & 91 & 87,5 & 1 & \\
\hline
\end{tabular}

Rev Bras Ginecol Obstet. 2006; 28(6): 331-9. 
foi inferior a 5 salários mínimos. A residência de um terço das gestantes era de alvenaria, as demais eram de madeira ou mistas e cerca de 3\% dos domicílios tinham esgoto a céu aberto. No terceiro trimestre de gestação $15,2 \%$ das gestantes do grupo de controle e $12,5 \%$ das do grupo intervenção referiram ser fumantes (Tabela 1).

A prevalência geral de anemia na amostra de gestantes foi de $28,6 \%$, sendo que $10,7 \%$ das gestantes apresentaram valores de hemoglobina inferiores a $10 \mathrm{~g} / \mathrm{dL}$. A freqüência de anemia no grupo intervenção foi de $31,6 \%$, não diferindo do observado no grupo controle, que foi $26,1 \%(p=0,433)$. O valor médio da hemoglobina no grupo intervenção na primeira avaliação foi $11,5 \pm 1,06 \mathrm{~g} / \mathrm{dL}$ e $11,6 \pm 11,39 \mathrm{~g} / \mathrm{dL}$ no segundo momento do estudo $(p=0,361)$. No último trimestre, $55,3 \%$ das gestantes do grupo intervenção apresentaram nivel de hemoglobina maior ou igual a $12 \mathrm{mg} / \mathrm{dL}$, e no grupo controle esse valor foi de 39, 1\% (sem diferença estatística).

Avaliando a adesão ao uso do suplemento nas 76 gestantes do grupo intervenção que completaram o estudo e receberam a prescrição de uso, observamos que $88,2 \%$ referiam usar o suplemento, sendo que dessas $67,7 \%$ fizeram uso diário, $24,9 \%$ não fizeram uso diário e $7,4 \%$ ingeriram a metade da dose prescrita. Entre as gestantes que usaram o suplemento, 67,7\% interromperam o uso em algum momento, sendo que os motivos citados com mais freqüência foram ter esquecido $(43,2 \%)$, enjôos e vômitos $(27,2 \%)$, cólica e diarréia $(9,1 \%)$ e outros $(20,5 \%)$. Essa interrupção durou em média $17,5 \pm 20,8$ dias. Entre as gestantes que fizeram uso do suplemento a aceitação foi referida por $65,7 \%$ das gestantes e os motivos citados para não aceitação foram: enjôo ou vômito (16) e cólica e/ou diarréia (7). No grupo controle o uso de algum tipo de suplemento de ferro foi referido por $66,3 \%$ das gestantes. A média de idade no inicio do uso foi de $18,5 \pm 7,6$ semanas de gestação e o tempo de uso variou de 4 a 32 semanas, com média de $13,6 \pm 8,3$ semanas.

Podemos observar na Tabela 2 que a freqüência de consumo dos alimentos estudados foi semelhante entre os grupos, sendo que 73,9\% das gestantes do grupo controle e $77,6 \%$ das do grupo intervenção consumiam carne diariamente, que o feijão fazia parte da refeição diária de mais de $60 \%$ das gestantes de ambos os grupos, que o pão e as massas foram consumidos diariamente por $89,1 \%$ das gestantes do grupo controle e por $94,7 \%$ das do grupo intervenção e que aproximadamente $18 \%$ das gestantes de ambos os grupos consumiam figado pelo menos uma vez na semana. O consumo diário de alimentos fontes de vitamina C foi semelhante entre os grupos, sendo de 60,9\% no grupo controle e 59,2\% no grupo intervenção; o consumo de alimentos fontes de cálcio foi superior a $70 \%$ para os dois grupos e os alimentos fontes de vitamina A estavam presentes em $22,8 \%$ das gestantes do grupo controle e em $11,8 \%$ no grupo intervenção. A média de consumo de pão e/ou massas entre todas as gestantes foi de 122,61 \pm 80,38 gramas por dia, que contribuiu em média com 3,08 $\pm 2,02 \mathrm{mg}$ de ferro. O ganho de peso também não mostrou diferença entre os grupos, sendo de aproximadamente de $12 \mathrm{~kg}$.

O consumo de algum tipo de bebida alcoólica foi referido por $16,7 \%$ das gestantes, sendo que $46,4 \%$ ingeriam em final de semana, e a ingestão de cerveja representou $78,6 \%$. O consumo de água foi referido por $95,8 \%$ das gestantes, a média de consumo foi de $1,5 \pm 0,7$ litros, sendo que $57,1 \%$ das gestantes ingeriam água de consumo impróprio.

A Tabela 3 apresenta as análises brutas e ajustadas para as variáveis estudadas em relação ao desfecho "anemia" para todas as gestantes estudadas. $\mathrm{Na}$ análise univariada a maior proporção de anemia entre as gestantes foi associada a baixa escolaridade, a maior concentração de pessoas por dormitório e tendência significativa para aquelas

Tabela 2 - Freqüência de consumo de alimentos entre gestantes do grupo controle e intervenção.

\begin{tabular}{|c|c|c|c|c|c|c|c|c|c|}
\hline \multirow{4}{*}{ Tipos de Alimentos } & \multicolumn{9}{|c|}{ Freqüência de consumo } \\
\hline & \multicolumn{4}{|c|}{ Grupo controle } & \multicolumn{4}{|c|}{ Grupo intervenção } & \multirow[t]{3}{*}{$p$} \\
\hline & \multicolumn{2}{|c|}{ Diário } & \multicolumn{2}{|c|}{ Semanal } & \multicolumn{2}{|c|}{ Diário } & \multicolumn{2}{|c|}{ Semanal } & \\
\hline & $n$ & $(\%)$ & $n$ & $(\%)$ & $\mathrm{n}$ & $(\%)$ & $\mathrm{n}$ & $(\%)$ & \\
\hline Alimentos fontes de cálcio & 68 & 73,9 & 24 & 26,1 & 54 & 71,1 & 22 & 28,9 & 0,679 \\
\hline Carnes & 68 & 73,9 & 24 & 26,1 & 59 & 77,6 & 17 & 22,4 & 0,576 \\
\hline Pães/Massas & 82 & 89,1 & 10 & 10,9 & 72 & 94,7 & 4 & 5,3 & 0,191 \\
\hline Feijões & 57 & 62,0 & 35 & 38,0 & 55 & 72,4 & 21 & 27,6 & 0,154 \\
\hline Folhas verdes escuras & 11 & 19,3 & 46 & 80,7 & 9 & 13,0 & 60 & 87,0 & 0,339 \\
\hline Alimentos fontes de vitamina $C$ & 56 & 60,9 & 36 & 39,1 & 45 & 59,2 & 31 & 40,8 & 0,827 \\
\hline Alimentos fontes de vitamina $A$ & 21 & 22,8 & 71 & 77,2 & 9 & 11,8 & 67 & 88,2 & 0,064 \\
\hline
\end{tabular}


Tabela 3 - Análise bruta e ajustada da presença de anemia e variáveis independentes entre gestantes.

\begin{tabular}{|c|c|c|c|c|c|}
\hline & \multicolumn{2}{|c|}{$\begin{array}{c}\text { Prevalência } \\
\text { hemoglobina } \\
<11 \text { g/dL }\end{array}$} & \multirow[t]{2}{*}{$\begin{array}{l}\text { OR bruto } \\
\text { IC (95\%) }\end{array}$} & \multirow[t]{2}{*}{$\begin{array}{l}\text { OR ajustado } \\
\text { IC }(95 \%)\end{array}$} & \multirow[t]{2}{*}{$p$} \\
\hline & $\mathrm{n}$ & $\%$ & & & \\
\hline \multicolumn{6}{|l|}{ Renda familiar } \\
\hline$<5$ salários mínimos & 42 & 32,3 & $2,4(0,9-6,3)$ & $1,5(0,5-4,2)$ & 0,41 \\
\hline$\geq 5$ salários mínimos & 6 & 16,2 & 1 & 1 & \\
\hline \multicolumn{6}{|l|}{ Escolaridade materna } \\
\hline$<8$ anos & 31 & 44,8 & $3,9(1,9-7,9)$ & $3,4(1,6-7,1)$ & 0,001 \\
\hline$\geq 8$ anos & 17 & 17,2 & 1 & 1 & \\
\hline \multicolumn{6}{|c|}{ Concentração por dormitório } \\
\hline$<3$ pessoas & 35 & 25,2 & 1 & 1 & \\
\hline$\geq 3$ pessoas & 13 & 44,8 & $2,4(1,0-5,5)$ & $1,9(0,7-4,6)$ & 0,14 \\
\hline \multicolumn{6}{|l|}{ Atividade da gestante } \\
\hline Não remunerada & 16 & 27,1 & $1,1(0,5-2,2)$ & - & - \\
\hline Remunerada & 32 & 29,2 & 1 & & \\
\hline \multicolumn{6}{|l|}{ Estado civil } \\
\hline Sem companheiro & 43 & 29,7 & $0,5(0,16-1,64)$ & & \\
\hline Com companheiro & 4 & 18,2 & 1 & - & - \\
\hline \multicolumn{6}{|l|}{ Idade } \\
\hline$<20$ anos & 12 & 35,3 & $1,4(0,6-3,3)$ & & \\
\hline$\geq 20$ anos & 36 & 26,9 & 1 & - & - \\
\hline \multicolumn{6}{|l|}{ Primípara } \\
\hline Sim & 14 & 25,0 & 1 & & \\
\hline Não & 34 & 30,4 & $1,3(0,6-2,7)$ & - & - \\
\hline \multicolumn{6}{|l|}{ Número de gestações } \\
\hline$\leq 3$ & 39 & 27,1 & 1 & 1 & \\
\hline$>3$ & 9 & 37,5 & $1,6(0,65-3,9)$ & $1,1(0,4-3,1)$ & 0,81 \\
\hline \multicolumn{6}{|l|}{ IMC pré-gestacional } \\
\hline$<19,8$ & 10 & 45,5 & $2,3(0,9-5,8)$ & $2,4(0,9-3,1)$ & \\
\hline$\geq 19,8$ & 36 & 26,3 & 1 & 1 & 0,07 \\
\hline \multicolumn{6}{|l|}{ Uso de Suplemento } \\
\hline Sim & 37 & 28,9 & 1 & 1 & \\
\hline Não & 11 & 27,5 & $0,9(0,4-2,0)$ & $0,8(0,3-2,3)$ & 0,76 \\
\hline \multicolumn{6}{|l|}{ Fumante } \\
\hline Sim & 8 & 27,6 & $0,9(0,3-2,3)$ & - & - \\
\hline Não & 40 & 28,8 & 1 & & \\
\hline \multicolumn{6}{|l|}{ Consumo de carne } \\
\hline$<P 75$ & 29 & 35,4 & $2,3(0,9-5,6)$ & $2,2(0,8-5,8)$ & \\
\hline$\geq \mathrm{P} 75$ & 8 & 19,0 & 1 & 1 & 0,08 \\
\hline \multicolumn{6}{|l|}{ Consumo de feijão } \\
\hline$<P 75$ & 21 & 37,5 & $1,3(0,6-2,9)$ & & \\
\hline$\geq \mathrm{P} 75$ & 17 & 30,9 & 1 & - & - \\
\hline \multicolumn{6}{|l|}{ Consumo de pão } \\
\hline$<\mathrm{P} 75$ & 27 & 27,3 & $0,7(0,3-1,6)$ & & \\
\hline$\geq \mathrm{P} 75$ & 26 & 32,0 & 1 & - & - \\
\hline \multicolumn{6}{|c|}{ Consumo de alimentos fonte de Vitamina C } \\
\hline Diário & 30 & 29,7 & 1 & & \\
\hline Não diário & 18 & 26,9 & $0,8(0,4-1,7)$ & - & - \\
\hline
\end{tabular}

Número 1 = categoria de referência; $\mathrm{OR}=$ odds ratio; IC $(95 \%)$ = intervalo de confiança a 95\%; IMC = índice de massa copórea.

Rev Bras Ginecol Obstet. 2006; 28(6): 331-9. 
com renda familiar inferior a cinco salários mínimos. Após regressão logística as gestantes com escolaridade inferior a 8 anos de estudo apresentaram 3 vezes mais risco para ocorrência de anemia.

A variável "número de gestações" superior a três", mostrou tendência para associação com o desfecho. A idade da gestante, IMC pré-gestacional, ser primigesta e número de consultas durante o pré-natal não foram associadas a anemia no presente estudo. Observou-se que, mesmo com a entrada das novas variáveis, a escolaridade da gestante inferior a oito anos permaneceu fortemente associada com o desfecho, sendo a única que permaneceu no modelo para participar da próxima etapa da regressão.

O consumo de carne inferior ao percentil 75 (200 gramas/dia) na análise bruta mostrou tendência para associação com anemia $\mathrm{OR}=2,32$ (0,95-5,68), no entanto o consumo de feijão, pão, vitamina $\mathrm{C}$, ser fumante e uso de suplemento durante a gestação não foram associados. A variável inclusa para a terceira fase da regressão foi consumo de carne e, apesar de não ter sido associada com anemia na análise bruta optou-se por manter a variável "uso de suplemento de ferro" no modelo, pois a mesma poderia ser fator de confusão. A variável escolaridade continuou a apresentar associação significativa mesmo com a introdução no último bloco de variáveis.

\section{Discussão}

A prevalência de $28,6 \%$ de anemia entre as gestantes, encontrada neste estudo, é inferior ao indice considerado de importância alarmante pela OMS, que é igual ou superior a 40\%. Prevalências entre 20,0 e $39,0 \%$ são consideradas de significância moderada no âmbito da saúde pública, sendo aceitos valores abaixo de $5 \%{ }^{1}$. Dessa forma os dados isolados encontrados no Brasil mostram que a situação da anemia durante a gestação está distante do desejado ${ }^{2-7}$.

As medidas para combater a deficiência de ferro e a anemia ferropriva estão bem estabelecidas, consistindo, resumidamente, em modificação dos hábitos alimentares; diagnóstico e tratamento das causas da perda crônica de sangue, controle de infecções e infestações que contribuem para a gênese e o agravamento da anemia, fortificação de alimentos e suplementação medicamentosa. O que ainda se discute é quanto à forma mais eficiente de realizar a suplementação com sais de ferro, devido à baixa resolutividade, seja pelo freqüente abando- no devido aos efeitos colaterais, seja pela reduzida absorção de ferro com os esquemas diários e de altas doses habitualmente prescritos ${ }^{17,18}$.

A análise entre o grupo controle da unidade de saúde e o grupo que recebeu intervenção dietética e uso padronizado de sulfato ferroso mostrou resultados semelhantes na prevalência de anemia entre os grupos. A investigação quanto ao uso de suplemento com ferro entre os grupos mostrou que $88,9 \%$ das gestantes do grupo intervenção referiram ter utilizado, sendo que no grupo controle essa freqüência foi de 66,3\%. Entretanto, a interrupção do uso foi observada em mais de $60 \%$ das gestantes do grupo intervenção. Na análise global multivaridada a referência ao uso ou não de suplemento não mostrou associação com o desfecho. Estudo realizado em Pernambuco mostrou resultados semelhantes aos nossos, em que a prevalência de anemia foi semelhante entre as gestantes que referiram fazer uso e aquelas que referiram não ter utilizado a suplementação durante a gestação ${ }^{19}$.

Verificou-se que na metade das unidades de saúde em Pernambuco não havia procedimentos técnicos padronizados (diagnóstico, prevenção e tratamento da anemia) e que esse fator pode contribuir com ocorrência de anemia entre gestantes ${ }^{20}$. No entanto, parece que mesmo na vigência desse procedimento padronizado não foi possivel melhorar o quadro de anemia na população estudada. No presente estudo, a análise de regressão mostrou que a variável mais fortemente associada à presença de anemia foi a escolaridade, exercendo papel fundamental no contexto da adesão às atitudes vinculadas ao adequado estado de saúde e nutrição. A menor renda familiar e a maior concentração de pessoas nos dormitórios mostraram tendência para associação. Esses resultados sugerem que sem mudanças estruturais nas condições socioeconômicas da população os programas de combate à anemia podem resolver parcialmente o problema, mas não permitirão que se alcancem as cifras recomendadas pela $\mathrm{OMS}^{1}$.

Durante a gestação ocorre aumento do requerimento de ferro, que passa de 1 a 2,5 mg/dia no primeiro trimestre para $6,5 \mathrm{mg} /$ dia no terceiro trimestre. A média de ingestão de ferro em países desenvolvidos é de 10 a $14 \mathrm{mg}$ por dia de ferro não heme, mas nem todo o elemento é absorvido. Evidência por meio de isótopos estáveis demonstrou que a porcentagem de ferro não-heme absorvido pela dieta durante a gestação aumentou de $7 \%$ na $12^{\mathrm{a}}$ semana para $36 \%$ na $24^{a}$ semana e $66 \%$ nas $36^{a}$ semana. Esses resultados demonstram que mulheres saudáveis são capazes de lidar com a alta demanda de ferro no periodo gestacional sem se tornarem anêmicas. 
Entretanto, para que isso aconteça as mulheres devem iniciar a gravidez com reservas adequadas ${ }^{21}$. É provável que a população estudada atendida no serviço público de saúde constitua grupo de risco para baixas reservas de ferro antes da gestação. O perfil alimentar avaliado não mostrou diferenças entre os grupos, confirmando a hegemonia quanto a esse aspecto. A análise com todas as gestantes mostrou tendência de associação entre maior consumo de carne e menor proporção de anemia. A ausência de significância pode ter ocorrido em função do tamanho amostral que não foi suficiente para essa análise.

Estudo no qual se compararam três esquemas de tratamento com sulfato ferroso durante a gestação revelou que no esquema diário houve fracasso de $2 \%$, e para os esquemas uma vez por semana e duas vezes por semana os fracassos foram de 41,6 e $13,2 \%$, respectivamente ${ }^{7}$. É importante destacar que as gestantes que não aderiram a $80 \%$ do estabelecido no programa foram excluídas do estudo. No presente trabalho, o objetivo foi avaliar a ocorrência de anemia e uso de suplemento de gestantes atendidas em unidade da rede básica de saúde, cuja proposta de prescrição foi suplementação diária de 60 mg. É conhecido que a ingestão diária é acompanhada de sintomas como náusea, vômitos, diarréia, constipação e dor abdominal, o que pode interferir na adesão ${ }^{17}$. Alguns investigadores advogam que, apesar de a suplementação diária ser mais eficaz, a suplementação intermitente é mais eficiente e com custo/beneficio melhor em programas de larga escala ${ }^{22}$. A adesão ao uso de suplemento é sem dúvida baixa, como foi demonstrado em estudo que analisou essa questão cuidadosamente ${ }^{23}$.

Existem críticas quanto ao uso isolado de suplemento de ferro para gestantes, pois se acredita que nos grupos populacionais de baixa renda, que são os alvos das politicas públicas, as deficiências de micronutrientes são mais abrangentes, requerendo portanto suplementos polivitamínicos e minerais

\section{Referências}

1. World Health Organization (WHO). Iron deficiency anaemia: assessment, prevention, and control. A guide for programme managers. Geneva;WHO; 2001.

2. Guerra EM, Barretto OCO, Vaz AJ, Silveira MB. Prevalência de anemia em gestantes de primeira consulta em centros de saúde de área metropolitana, Brasil. Rev Saúde Pública. 1990;24(5):380-6.

3. Fujimori E, Laurenti D, Núñez De Cassana LM, Oliveira IMV, Szarfarc SC. Anemia e deficiência de ferro em gestantes adolescentes. Rev Nutr. 2000;13(3):177-84.

4. Sinisterra Rodriguez O, Szarfarc SC, Benicio MHA. Anemia e desnutrição maternas e sua relação com o peso ao nascer. Rev Saúde Pública. 1991;25(3):193-7. para reduzir a prevalência da prematuridade e baixo peso ao nascer. Entretanto, estudo randomizado duplo-cego desenvolvido no México não demonstrou diferenças nessas prevalências entre grupo de gestantes que receberam suplementos completos de micronutrientes e aquelas que usaram suplementos contendo apenas o ferro ${ }^{24}$.

Estudos clínicos randomizados forneceriam melhores respostas quanto ao impacto da suplementação de ferro. Entretanto, a maior limitação é baseada nos termos éticos, já que esse tipo de estudo exigiria um grupo controle que não recebesse o tratamento padrão, já que muitos países já adotam como rotina a suplementação de ferro para gestantes, em função do aumento do requerimento desse nutriente conforme recomendações internacionais da $\mathrm{OMS}^{1}$. Assim, sugere-se que estudos prospectivos sejam realizados em países em desenvolvimento que enfoquem o impacto do uso do suplemento e do padrão dietético no prognóstico da gestação e indicadores bioquímicos ${ }^{25}$.

As conclusões deste estudo confirmam que, apesar da recomendação de que todas as gestantes devam receber suplementação com ferro, outras medidas urgentes devem ser implementadas para que as cifras de anemia ferropriva entre gestantes alcancem valores de países desenvolvidos, especialmente quanto à melhora no acesso à educação. O país já avançou muito na prevenção da anemia ferropriva, já que estabeleceu como politica pública a fortificação obrigatória das farinhas com ferro e ácido fólico, propiciando às mulheres em idade fértil iniciarem a gestação com melhores reservas de ferro. Com relação à nova política nacional de suplementação universal para todas as gestantes com suplementação de $60 \mathrm{mg}$ de ferro e $5 \mathrm{mg}$ de ácido fólico que já está sendo implementada pelo Ministério da Saúde, enfatiza-se a importância de avaliação sistemática do seu impacto na prevalência de anemia ferropriva no curto e médio prazo.

5. Bergmann RL, Gravens-Muller L, Hertwig K, Hinkel $\mathrm{J}$, Andres B, Bergmann KE, et al. Iron deficiency is prevalent in a sample of pregnant women at delivery in Germany. Eur J Obstet Gynecol Reprod Biol. 2002;102(2):155-60.

6. Papa ACE, Furlan JP, Pasquelle M, Guazzelli CAF, Figueiredo MS, Camano L, et al. A anemia por deficiência de ferro na grávida adolescente: comparação entre métodos laboratoriais. Rev Bras Ginecol Obstet. 2003;25(10):731-8.

7. Souza AI, Batista Filho M, Ferreria LOC, Figueirôa JN. Efetividade de três esquemas com sulfato ferroso para tratamento de anemia em gestantes. Rev Panam Salud Pública. 2004;15(5):313-9.

Rev Bras Ginecol Obstet. 2006; 28(6): 331-9. 
8. Conde-Agudelo A, Belizan JM, Lindmark G. Maternal morbidity and mortality associated with multiple gestations. Obstet Gynecol. 2000;95(6 Pt 1):899-904.

9. Kulier R, de Onis M, Gulmezoglu AM, Villar J. Nutritional interventions for the prevention of maternal morbidity. Int J Gynaecol Obstet. 1998;63(3):231-46.

10.Scholl TO, Hediger ML, Fischer RL, Shearer JW. Anemia vs iron deficiency: increased risk of preterm delivery in a prospective study. Am J Clin Nutr. 1992;55(5):985-8.

11.Quillan, JP, Kwong A, Passmore P. An epidemiological investigation of pre-eclampsia and elevated blood pressure among Kampuchean refugee women at Sakaeo Holding Center, Thailand. J Trop Med Hyg. 1983;86(5):185-91.

12. Spinillo A, Capuzzo E, Piazzi G, Nicola S, Colonna L, Iasci A. Mage infants. Early Hum Dev. 1994;38(1):35-43.

13.Frederick IO, Rudra CB, Miller RS, Foster JC, Williams MA. Adult weight change, weight cycling, and prepregnancy obesity in relation to risk of preeclampsia. Epidemiology 2006;17(4):428-34.

14.Aquino MMA, Cecatti JG, Mariani Neto C. Risk factors associated to fetal death. São Paulo Med J. 1998;116(6):1852-7.

15.Neilson JP. Maternal mortality. Curr Obstet Gynaecol. $2005 ; 15(6): 375-81$.

16.Institute of Medicine (IOM). Nutrition during pregnancy. Weight gain. Nutrient supplements. Washington: National Academic Press; 1992.

17. Batista Filho M, Ferreira LOC. Prevenção e tratamento da anemia nutricional ferropriva: novos enfoques e perspectivas. Cad Saúde Pública. 1996;12(3):411-5.

18. Hallberg L, Ryttinger L, Solvell L. Side-effects of oral iron therapy. A double-blind study of different iron compounds in tablet form. Acta Med Scand Suppl. 1996; 459:3-10.

19.Pereira RC. Anemia em parturientes da Maternidade Prof. Monteiro de Moraes e peso ao nascer: impacto do condicionantes macro, e micro-estruturas [tese]. Recife: Universidade Federal de Pernambuco; 1997.

20.Santos ICRV, Batista Filho M. Anemia no atendimento pré-natal em Pernambuco. Recife: Liceu; 2001. p. 79-80.

21.Barrett JF, Whittaker PG, Williams JG, Lind T. Absorption of non-haem iron food during normal pregnancy. BMJ. 1994;309(6947):79-82.

22.Schultink W, Gross R. Use of daily compared with weekly iron supplementation: apples and pears. Am J Clin Nutr. 1999;69(4):739-42.

23.Ekstrom EC, Kavishe FP, Habicht JP, Frongillo EA Jr, Rasmussen KM, Hemed L. Adherence to iron supplementation during pregnancy in Tanzania: determinants and hematologic consequences. Am J Clin Nutr. 1996;64(3):368-74.

24.Ramakrishan U, Neufeld LM, Gonzalez-Cóssio T, Villalpando S, Garcia-Guerra A, Rivera J, et al. Multiple micronutrient supplements during pregnancy do not reduce anemia or improve iron status compared to iron-only supplements in Semirural México. J Nutr. 2004;134(4):898-903.

25.Ramakrishnan U, Martorell R, Schoroeder DG, Flores $\mathrm{R}$. Role of intergenerational effects on linear growth. J Nutr. 1999; 129(2S Suppl):544S-549S. 